American Medical Journal 3 (2): 240-248, 2012

ISSN 1949-0070

(C) 2012 Science Publications

\title{
The Role of Inflammation in Development and Therapy of Malignant Mesothelioma
}

\author{
Jill Miller and Arti Shukla \\ Department Pathology, College of Medicine, \\ University of Vermont, 89 Beaumont Avenue, Burlington, VT
}

\begin{abstract}
Malignant Mesothelioma (MM) is an asbestos related malignancy with a poor prognosis and limited therapeutic approaches. The pathogenesis of $\mathrm{MM}$ has been linked to asbestos induced inflammation. Asbestos exposure results in reactive oxygen species generation, infiltration of inflammatory cells and prolonged release of multiple cytokines, oxidants and growth factors. The role of inflammation in MM has led to the evaluation of inflammatory profiles as prognostic and therapeutic markers. Additionally, inflammatory pathways are under investigation for potential therapeutic interventions. In this review, we discuss the role of inflammation in MM pathogenesis, inflammatory markers with potential clinical impact for $\mathrm{MM}$ and clinical trials that target inflammatory pathways and responses for treatment of MM. Ultimately, MM remains a difficult to treat cancer that requires multimodality therapy.
\end{abstract}

Key words: Best Investigator's Choice, Malignant Mesothelioma (MM), Reactive Oxygen Species, Malignant Pleural Mesothelioma, Extrapleural Pneumonectomy

\section{INTRODUCTION}

Malignant Mesothelioma (MM) is caused by asbestos and produces devastating tumor types with poor prognosis and no effective therapeutic approaches. Asbestos exposure is known to increase the risk of pulmonary pathology in the form of nonmalignant inflammatory diseases, such as pleural plaques, pleural effusions and asbestosis and malignant diseases such as mesothelioma and bronchogenic carcinoma. The differences in the cellular phenotype involved (alveolar, epithelial, or mesothelial cells) defines the outcome of disease. Asbestos-induced inflammatory changes may, in part, be responsible for diseases. Pleural plaque is produced by the effect of recurrent inflammatory and repair processes occurring for long time periods. Chronic inflammatory episodes may predispose to malignant evolution, as it is known that the majority of MM develops on pleura affected by pleural plaques and not on the normal pleura. A survey of the current literature suggests that there is more than one mechanism involved in the pathogenesis of asbestosinduced mesothelioma. One proposed mechanism is the oxidative stress concept that highlights how iron within asbestos fibers catalyzes free radical generation and thereby induces oxidative stress and carcinogenesis (Shukla et al., 2003). Another proposed mechanism is the chronic inflammatory theory that is founded on the observation that inflammation is a biologic response to pathogenic materials and injured cells in which various types of cells, such as neutrophils, macrophages, fibroblasts and vascular endothelial cells, interact with each other. Over time, inflammation becomes chronic and plays an important role in asbestos-induced carcinogenesis that is characterized by persistent release of cytokines and oxidants from macrophages. One way proinflammatory cytokines promote carcinogenesis in epithelial and mesothelial cells is by altering signaling pathways and thereby inhibiting apoptosis. In addition, persistent macrophage activation plays important roles in initiation, as well as promotion, of pathological processes in mesothelioma. The remaining mechanisms include the chromosome tangling concept, which postulates that asbestos fibers damage chromosomes when cells divide and the adsorption concept which states that the surface of asbestos possesses a high affinity for certain molecules including components of cigarette smoke as well as endogenous molecules. In the present review, we focus on the role of inflammation in development and therapy of MM.

Asbestos exposure related inflammatory changes leading to malignant mesothelioma: Asbestos exposure to cells and tissues results in Reactive Oxygen Species (ROS) generation that triggers lipid peroxidation, leading to inflammation related gene

Corresponding Author: Arti Shukla, Department Pathology 216 HSRF, College of Medicine, University of Vermont, 89 Beaumont Avenue, Burlington, VT Tel: 802-656-8253 Fax: 802-656-8892 
expression (Janssen-Heininger et al., 2000; Shukla et al., 2003). Our group has repeatedly demonstrated the role of asbestos in inflammation. Using mouse asbestosinhalation models, we have shown inflammation in terms of altered levels of cytokines, differential cell counts in bronchoalveolar lavage fluid (BALF) (SaboAttwood et al., 2005; Shukla et al., 2007) and myeloperoxidase status in lung neutrophils (Haegens et al., 2005). In addition, Ogami et al. (2004) demonstrated an asbestos-induced inflammatory condition by morphometric analysis of rat lungs. In their experiment, rats were exposed to an asbestos dose comparable to asbestos levels detected in work environments (Ogami et al., 2004). It has been reported by many groups that the highest level of inflammation likelyoccurs at the site of fiber deposition. Pleural mesothelium function is possibly altered either directly or indirectly by the exposure of the lung to asbestos (Adamson, 1997). The growth phase of mesothelial cells in visceral pleura is very early and unrelated to pleural fiber deposition in an animal model of asbestos exposure. This process was found to depend on cytokine release by cells in the lung.

Although acute inflammatory responses in the lung parenchyma constitute an important defense mechanism in normal circumstances, chronic inflammation is considered to be an important contributor to tissue damage, inducing lung pathologies including mesothelioma. Fiber-induced inflammation in the parenchyma reverses both the normal flow of lymph and the normal transpleural pressure, resulting in a net flow of fluid and fibers directly into the pleural space from the underlying parenchyma (Miserocchi, 1997). This leads to mesothelial and endothelial cell damage, inflammation and the accumulation of pleural macrophages. Pleural macrophages undergo frustrated phagocytosis in an attempt to enclose the long fibers. The process of frustrated phagocytos is induces prolonged release of cytokines and oxidants that ultimately lead to further inflammation, fibrosis and genotoxicity in bystander mesothelial cells. Increased pathogenicity of long asbestos fibers depends on the persistent presence of fibers, repeated fiber-induced injury, tissue repair and local inflammation (Moalli et al., 1987; Donaldson et al., 1989).

As discussed below, there are numerous studies supporting the concept that there is an inflammatory reaction following asbestos exposure (Choe et al., 1997). Hill et al. (2003) reported that amosite asbestos causes pleural inflammation by increased secretion of Intercellular Adhesion Molecule-1 (ICAM-1), Monocyte Chemoattractant Protein-1 (MCP-1) and Macrophage Inhibitory Protein-2 (MIP-2) in pleural lavage fluid as well as in vitro mesothelial cell culture (Hill et al., 2003). Recent studies from our group have also shown that human mesothelial cells acquire resistance to asbestos-induced toxicity via induction of one or more Epidermal Growth Factor Receptor (EGFR)-linked survival pathways (PI3K/AKT/ERK1/2) during Simian Virus 40 (SV40) transformation and carcinogenesis (Shukla et al., 2011). Many cytokines and growth factors are shown to be implicated in asbestos-induced MM pathogenesis including Tumor Necrosis Factor alpha (TNF- $\alpha$ ), Transforming Growth Factor Beta (TGF- $\beta$ ), Platelet Derived Growth Factor (PDGF), Insulin like Growth Factor (IGF) (Liu and Klominek, 2004), interleukin-6 (IL-6), interleukin-8 (IL-8) (Galffy et al., 1999), Vascular Endothelial Growth Factor (VEGF) (Strizzi et al., 2001) and Hepatocyte Growth Factor (HGF) (Cacciotti et al., 2001). TNF- $\alpha$ is released in response to large accumulations of macrophages undergoing phagocytosis of asbestos. The binding of the released TNF- $\alpha$ to its receptor, TNF-R1, which is also expressed by mesothelial cells and activated by NFאB pathway, increases the percentage of human mesothelial cells that survive asbestos exposure (Yang et al., 2006). Our recent in vitro and in vivo studies also support the role of asbestos in inflammation-induced mesothelioma. We recently reported, using Affymetrix microarray and BioPlex analysis in primary and telomerase immortalized human mesothelial cells, that asbestos exposure causes increased inflammatory responses that include IL-1 $\beta$, IL-13, basic Fibroblast Growth Factor (bFGF), VEGF and granulocyte colony stimulating factor (G-CSF) which may be responsible for mesotheliomagenic transformation of these cells (Shukla et al., 2009; Hillegass et al., 2010a). In addition, our in vivo work confirms that MM development in intraperitoneal mouse models is preceded by increased levels of many of these cytokines and growth factors (Hillegass et al., 2010b).

Elegant work by our group in collaboration with others has demonstrated, for the first time, that the NOD-Like Receptor Protein 3 (NLRP3) inflammasome plays a critical role in asbestos inhalation-induced inflammation in mouse lungs (Dostert et al., 2008). Further studies in human mesothelial cells confirmed that asbestos exposure caused priming of NLRP3 (increased mRNA levels) as well as its activation (Shukla et al., 2003; 2007; 2009; 2011). In support of our work, Yang et al. (2010) showed the release of high mobility group box-1 protein (HMGB-1) from asbestosexposed mesothelial cells (Yang et al., 2010). The release of HMGB-1 may possibly occur via inflammasome-induced pyroptosis, an inflammation- 
mediated cell death process dependent on inflammsome activation of caspase-1. Additionally, a recent study has reported the ability of the iron in Libby amphibole to induce the inflammasome cascade (Shannahan et al., 2012).

Radiation can also induce mesothelioma in animals and humans and may be linked to inflammation. It has been reported by many groups that cancer patients with lymphoma who received radiotherapy were at high risk of developing mesothelioma as long as 21 years after radiation exposure (Brown et al., 2006; Teta et al., 2007). Radiation to the abdomen and thorax has also been reported to result in MM development (Amin et al., 2001; Travis et al., 2005). In a rare case of pericardial mesothelioma, the authors considered inflammation and healing, as a result from a pericardiotomy, to have a synergistic effect with asbestos in the pathogenesis of the tumor (Rizzardi et al., 2010).

Inflammatory prognostic and therapeutic markers: Several studies have shown that inflammatory profiles in tumors can be used as prognostic as well as therapeutic markers. Interleukin-6 (IL-6) is a multifunctional cytokine that regulates immune response and inflammation and its overproduction has been shown to underlie a number of malignancies including mesothelioma. Blocking of IL-6 signaling may be a therapeutic possibility in mesothelioma (Nishimoto, 2010). Another interleukin under investigation for prognostic and therapeutic potential in MM is interleukin-4 (IL-4). Burt et al. (2012) assessed tumor expression of interleukin-4 receptor $\alpha$ (IL-4R $\alpha$ ) by RT-PCR analysis in 37 Malignant Pleural Mesothelioma (MPM) specimens from patients undergoing surgical resection. Greater expression of IL$4 \mathrm{R} \alpha$ was predictive of poor survival in epithelial, but not non-epithelial subtypes of MPM patients. Additionally, the study showed that $\mathrm{T}$ cells within MPM tumors from eight patients undergoing Extrapleural Pneumonectomy (EPP) produced higher frequencies of IL-4 upon stimulation compared to matched $\mathrm{T}$ cells from blood samples. Furthermore, human MPM cells had increased STAT-6 phosphorylation and increased production of inflammatory cytokines, IL-6, IL-8 and VEGF, in response to IL-4 (Burt et al., 2012).

Based on research supporting asbestos-induced inflammation in the pathogenesis of MM, Kao et al. (2010; 2011) investigated the blood neutrophil-tolymphocyte ratio (NLR), an index of systemic inflammation, as a prognostic factor in MM patients (Kao et al., 2010). The findings from the study indicated that the NLR is an independent predictor of survival for patients with MM undergoing systemic therapy. A separate study with 85 patients demonstrated that both low calretinin expression and high NLR were independently associated with poor prognosis in patients with MPM undergoing EPP (Kao et al., 2011). Pinato et al. (2012) validated the prognostic role of NLR in a large cohort of 171 MPM patients and also found the modified Glasglow Prognostic Score (mGPS), based on levels of $\mathrm{C}$ reactive protein and hypoalbuminemia, to be an independent predictor of survival by multivariate analysis (Pinato et al., 2012).

The prognostic value of tumor-infiltrating lymphocytes on survival of patients with MPM has been studied in small patient series at single institutions (Leigh and Webster 1982; Yamada et al., 2010). Anraku et al. (2008) analyzed 32 specimens from MPM patients who were treated with induction chemotherapy followed by EPP for distribution of $T$ cells by immunohistochemical analysis. They demonstrated that the presence of high levels of CD8+ tumor-infiltrating lymphocytes was associated with improved prognosis, lower incidence of mediastinal node disease and longer progression free survival (Anraku et al., 2008). In another study, Yamada et al. (2010) investigated tumorinfiltrating lymphocytes by immunohistochemistry in MPM specimens and analyzed a subpopulation of 27 patients who underwent surgical resection. It was found that these patients showed a significantly better prognosis in correlation with a high density of CD8+ tumor-infiltrating lymphocytes (Yamada et al., 2010). A semi-quantitative assessment of inflammatory response on routine hematoxylin and eosin stained slides can also predict survival in patients with epithelioid MPM. A study on 175 epithelioid MPM specimens investigated inflammatory responses in tumors and stroma and suggested a positive prognostic value of inflammatory response in the stroma of epithelioid MPM. Interestingly, inflammation within the tumor, as opposed to the stroma, was associated with a vascular invasion, a poor prognostic feature (Suzuki et al., 2011). Collectively, these studies provide rationale for investigating immunotherapy to benefit epithelioid MPM patients.

HMGB-1, a damage associated molecular pattern protein that is involved in asbestos induced inflammation, has been demonstrated to be present in higher levels in the serum of $20 \mathrm{MM}$ patients compared to 20 age-and-gender matched healthy controls (Jube and Rivera, 2012). In the same study, HMGB-1 was analyzed in $31 \mathrm{MM}$ biopsies and cytoplasmic staining of HMGB-1 was shown to correlate with tumor stage, however this correlation needs to be validated due to small sample size. Additionally, inhibition of HMGB-1 in vivo by anti-HMGB-1 neutralizing antibodies 
suppressed MM xenographs from specific, but not all MM cell lines evaluated. Together, the above findings support the need for further investigation of the HMGB-1 axis as a potential prognostic and therapeutic target for MM in the future.

Conventional therapy of malignant mesothelioma: Surgical resection is the only curative treatment of MPM while other treatments minimally improve response rate and overall survival. The majority of patients diagnosed with MPM are unable to undergo surgical resection because of advanced disease at time of presentation. The standard of care for these patients is chemotherapy with cisplatin plus an antifolate, such as pemetrexed or raltitrexed, which improves overall survival from 9 months to 12 months (Vogelzang et al., 2003; Kelly et al., 2011). Radiotherapy may be used for palliative care, however there is no evidence for routine use of radiation as primary therapy for MPM (Price, 2011). For patients with surgically resectable MPM, surgical options include extrapleural pneumonectomy or pleurectomy with decortication which may be combined with intracavitary chemotherapy at time of resection or systemic chemotherapy and radiotherapy (Richards et al., 2006; Takigawa et al., 2011). Unfortunately, only a small percentage of MPM patients will qualify for multimodality treatment and despite this aggressive therapy, MM recurrence is frequent.

Diffuse malignant peritoneal mesothelioma (DMPM) makes up 15-20\% of MM diagnoses and like MPM, is typically diagnosed at late stage (Turner et al., 2012). DMPM is ultimately fatal although advances have been made in therapeutic strategies for surgically resectable disease. The combination of cytoreductive surgery and hyperthermic intraperitoneal chemotherapy with cisplatin plus doxorubicin or mitomycin-C has been shown to improve overall survival (Baratti et al., 2011; Turner et al., 2012). Patients with inoperable DMPM may undergo systemic chemotherapy with cisplatin and pemetrexed and/or palliation surgery.

Experimental therapy of malignant mesothelioma: Inflammation presents a new avenue for the exploration of therapeutic interventions for MM. Novel therapies such as gene therapy, immunotherapy and targeted molecular therapy are under investigation through preclinical research and clinical trials (Takigawa et al., 2011; Vachani et al., 2011; Zauderer and Krug, 2012). Here we review clinical trials that target modulation of inflammatory responses in MM treatment.

MM expresses high levels of Epidermal Growth Factor Receptor (EGFR) however clinical trials have shown limited therapeutic activity of EGFR inhibitors in MM patients. A phase II clinical trial investing gefitinib, a selective inhibitor of EGFR, demonstrated this agent was not effective in the treatment of MM and overexpression of EGFR did not predict response to gefitinib (Govindan et al., 2005). Other tyrosine kinase inhibitors, such as imatinib and erlotinib, have been studied in phase II clinical trials and have produced negative results (Mathy et al., 2005; Garland et al., 2007). This includes a multicenter, phase II clinical trial that investigated erlotinib in combination with bevacizumab, a VEGF inhibitor, in previously treated MPM patients and showed no complete or partial responses (Jackman et al., 2008). Postulated resistance mechanisms to EGFR inhibitors include activation of downstream pathways of Extracellular signal Regulated Kinase (ERK) and phosphatidylinositol 3-kinase/Akt and absence of mutations in EGFR kinase domain (Garland et al., 2007; Velcheti et al., 2009). Ongoing clinical trials involving inhibition of EGFR include a multicenter, phase II study of Cetuximab, a monoclonal antibody against EGFR, in combination with standard first-line chemotherapy in MPM patients (Clinicaltrials.gov identifier: NCT0996567) and phase II study of erlotinib in malignant peritoneal mesothelioma patients who harbor EGFR kinase domain mutations (Clinicaltrials.gov identifier: NCT01592383).

Growth factors under MM clinical investigation include Insulin-Like Growth Factor (IGF) and HGF with downstream pathways involved in cell growth, anti-apoptosis, invasion and metastasis (Klominek et al., 1998; Zha and Lackner 2010). Cixutumumab, a monoclonal antibody to Insulin-like Growth Factor receptor 1 (IGF-IR), has been shown to delay growth of mesothelioma tumor xenographs and improve overall survival in mice (Kalra et al., 2012). A phase II clinical trial is evaluating IMC-A12 (cixutumumab) in pleural and peritoneal MM patients who have failed treatment with chemotherapy. The primary outcome is clinical response rate (Clinicaltrials.gov identifier: NCT01160458). Another phase II clinical trial is investigating an Anti-HGF Monoclonal antibody, (AMG) 102, in combination with pemetrexed disodium and cisplatin in MPM patients with a primary outcome of progression free survival (Clinicaltrials.gov identifier: NCT01105390).

Tumor-homing peptide asparagine-glycinearginine (NGR)-human tumor necrosis factor alpha (NGR-hTNF) is being investigated for its role to induce antitumor activity in MM patients. This agent is a modification of TNF- $\alpha$ to reduce systemic toxicity and selectively bind aminopeptidase $\mathrm{N}$ isoform which is overexpressed on tumor blood vessels (Gregorc et al., 2010). A multicenter, nonrandomized, single agent, phase II clinical trial in previously treated MM patients 
showed NGR-hTNF was tolerated and resulted in disease control in about half the patients (Gregorc et al., 2010). In a randomized, double blinded, phase III clinical trial of NGR-hTNF plus Best Investigator's Choice (BIC) versus placebo plus BIC was initiated in MPM patients who have progression of disease after treatment with pemetrexed-based chemotherapy. Overall survival is the primary outcome measure and the estimated completion of the study is February of 2013 (Clinicaltrials.gov identifier: NCT01098266). NGR-hTNF is also being evaluated as maintenance therapy in MM. A randomized, double blinded, phase II clinical trial is investigating the efficacy of NGR-hTNF plus best supportive care versus placebo plus best supportive care as maintenance therapy in advanced MPM patients with non-progressing disease immediately following six cycles of pemetrexed-based chemotherapy. The primary end point is progression free survival and the total enrollment is expected to be 100 patients (Clinicaltrials.gov identifier: NCT01358084).

Interferons are immunostimulatory cytokines that enhance anti-tumor responses. Clinical trials involving gene therapy in $\mathrm{MM}$ patients that aimed to deliver interferon beta (IFN- $\beta$ ) through adenoviral vectors by intrapleural administration have shown to be feasible and resulted in induction of humoral immune responses and positive clinical responses. However, the development of neutralizing antibodies remains a limitation for this therapeutic method (Sterman et al., 2007; 2010). Other ongoing clinical trials involving interferon therapy and MM include a phase I clinical trial investigating the gene transfer of IFN- $\alpha$ (Clinicaltrials.gov identifier: NCT01212367) and a phase 0 clinical trial evaluating intrapleural adenovirus mediated IFN- $\alpha$ in combination with first-line or second-line chemotherapy (Clinicaltrials.gov identifier: NCT01119664).

TGF- $\beta$ is a multifunctional cytokine that is overexpressed in $\mathrm{MM}$ and may function as a tumor promoter through immunosuppression. TGF- $\beta$ has received attention as a therapeutic target based on in vivo studies that demonstrated blocking of TGF- $\beta$ by neutralizing antibodies, TGF inhibitors and other methods resulted in MM tumor inhibition (Marzo et al., 1997; Suzuki et al., 2004; 2007). A phase II clinical trial investigating the use of an anti-TGF monoclonal antibody, GC1008, in relapsed MPM patients is in progress. The primary outcome is assessment of survival rate progression at 3 months (Clinicaltrials.gov identifier: NCT01112293). Activin like receptor kinase 1 (ALK-1) is a member of the TGF- $\beta$ type I receptor family and is involved in angiogenesis (Oh et al., 2000). An ongoing phase II clinical trial is investigating an anti-ALK-1 monoclonal antibody, PF-03446962, in advanced stage MPM patients. Response rate is the primary outcome and estimated completion of the study is June 2014 (Clinicaltrials.gov identifier: NCT01486368).

IL-2 is a proinflammatory cytokine and promotes development of cytotoxic T lymphocytes. Past clinical trials have shown that intrapleural administration of IL2 , as opposed to subcutaneous and intravenous route, has the greatest potential for anti-tumor activity with acceptable toxicity when administered alone or in combination with multimodality therapy (Astoul et al., 1998; Castagneto et al., 2001; Mulatero et al., 2001; Lucchi et al., 2007). Ali et al. (2009) conducted a clinical trial that demonstrated intrapleural preoperative IL-2 treatment in 60 MPM patients resulted in increased tryptase mast cells and tumor infiltrating lymphocytes compared to 30 untreated MPM patients undergoing surgical resection. Additionally, the study proposed that the above immunological parameters may be used as predictors of outcome in MPM patients treated with IL2 (Ali et al., 2009).

Mesothelin has become the target for antibodies against $\mathrm{MM}$ as this cytoplasmic membrane glycoprotein is highly expressed in epithelioid subtype MM tumors with limited expression on normal human tissues (Kelly et al., 2011). Currently, there are multiple anti-mesothelin antibodies under investigation in clinical trials for the treatment of MM. MORAb-009 is a high affinity monoclonal antibody for mesothelin that is implicated to disrupt the cell adhesion function of mesothelin and induce cell mediated cytotoxicity (Hassan et al., 2007). A phase I clinical trial was conducted in patients with high mesothelin expressing advance solid tumors which included 13 MM patients out of 24 patients enrolled. The study demonstrated MORAb-009 was safe and well tolerated. Of 20 patients who completed at least one cycle of MORAb009, 11 had stable disease although no objective clinical responses were observed (Hassan et al., 2010). An open label, phase II clinical trial is now in place which investigates MORAb-009 in combination with cisplatin and pemetrexed in patients diagnosed with epithelioid or biphasic mesothelioma subtypes without prior systemic chemotherapy or radiotherapy. The primary outcome is progression free survival (Clinicaltrials.gov identifier: NCT 00738582). SS1P is a recombinant immunotoxin made up of an antimesothelin $\mathrm{Fv}$ fragment linked to a truncated Pseudomonas exotoxin (Chowdhury and Viner, 1998). A single center, phase I clinical trial evaluating dose escalation of SS1P with concurrent administration of cisplatin and pemetrexed in unresectable epithelioid MPM patients is underway (Clinicaltrials.gov 
identifier: NCT01445392). Additionally, SS1P is being investigated in a phase I clinical trial in mesothelioma patients with immune suppression drugs, pentostatin and cyclophosphamide, as an attempt to decrease the immunogenicity of SS1P (Clinicaltrials.gov identifier: NCT01362790).

\section{CONCLUSION}

MM is a difficult to treat cancer associated with asbestos exposure and requires a multidisciplinary approach such as the combination of surgery, chemotherapy and radiation. It is not realistic to expect that any single intervention will be enough to treat this aggressive cancer. Inflammation plays an important role in $\mathrm{MM}$ development and improving our understanding of this phenomenon will help identify inflammatory biomarkers that are useful predictive or prognostic tools as well as facilitate the development of novel treatments for this deadly disease.

\section{REFERENCES}

Adamson, I.Y., 1997. Early mesothelial cell proliferation after asbestos exposure: in vivo and in vitro studies. Environ. Health Perspect., 5: 12051208. PMID: 9400724

Ali, G., L. Boldrini, M. Lucchi, A. Picchi and M.C. Prati, et al., 2009. Treatment with interleukin-2 in malignant pleural mesothelioma: immunological and angiogenetic assessment and prognostic impact. Br. J. Cancer., 101: 1869-1875. PMID: 19935800

Amin, A.M., C. Mason and P. Rowe, 2001. Diffuse malignant mesothelioma of the peritoneum following abdominal radiotherapy. Eur. J. Surg. Oncol., 27: 214-215. PMID: 11289763

Anraku, M., K.S. Cunningham, Z. Yun, M.S. Tsao and L. Zhang et al., 2008. Impact of tumor-infiltrating $\mathrm{T}$ cells on survival in patients with malignant pleural mesothelioma. J. Thorac. Cardiovasc Surg., 135: 823-829. PMID: 18374762

Astoul, P., D. Picat-Joossen, J.R. Viallat, C. Boutin et al. 1998. Intrapleural administration of interleukin2 for the treatment of patients with malignant pleural mesothelioma: A Phase II study. Cancer, 83: 2099-2104. PMID: 9827714

Baratti, D., S. Kusamura and M. Deraco, 2011. Diffuse malignant peritoneal mesothelioma: systematic review of clinical management and biological research. J. Surg. Oncol., 103: 822-831. DOI: $10.1002 /$ jso. 21787
Brown, L.M., R.A. Howard and L.B. Travis, 2006. The risk of secondary malignancies over 30 years after the treatment of non-Hodgkin lymphoma. Cancer, 107: 2741-2742. PMID: 17039496

Burt, B.M., A. Bader, D. Winter, S.J. Rodig, R. Bueno and D.J. Sugarbaker, 2012. Expression of interleukin-4 receptor alpha in human pleural mesothelioma is associated with poor survival and promotion of tumor inflammation. Clin. Cancer Res., 18: 1568-1577. PMID: 22261806

Cacciotti, P., R. Libener, P. Betta, F. Martini and C. Porta et al., 2001. SV40 replication in human mesothelial cells induces HGF/Met receptor activation: a model for viral-related carcinogenesis of human malignant mesothelioma. Proc. Natl. Acad Sci. USA., 98: 12032-12037. DOI: 10.1073/pnas.211026798

Castagneto, B., S. Zai, L. Mutti, A. Lazzaro and R. Ridolfi et al., 2001. Palliative and therapeutic activity of IL-2 immunotherapy in unresectable malignant pleural mesothelioma with pleural effusion: Results of a phase II study on 31 consecutive patients. Lung. Cancer, 31: 303-310. PMID: 11165411

Choe, N., S. Tanaka, W. Xia, D.R. Hemenway, V.L. Roggli and E. Kagan, 1997. Pleural macrophage recruitment and activation in asbestos-induced pleural injury. Environ. Health Perspect, 105: 1257-1260.

Chowdhury, P.S. and J.L. Viner, 1998. Isolation of a high-affinity stable single-chain Fv specific for mesothelin from DNA-immunized mice by phage display and construction of a recombinant immunotoxin with anti-tumor activity. Proc. Natl. Acad Sci. USA., 95: 669-674. Donaldson, K., G. M. Brown, D.M. Brown, R.E. Bolton and J.M. Davis, 1989. Inflammation generating potential of long and short fibre amosite asbestos samples. Br. J. Ind. Med., 46: 271-276. PMID: 2540793

Dostert, C., V. Petrilli, R.V. Bruggen, C. Steele and B.T. Mossman et al., 2008. Innate immune activation through Nalp3 inflammasome sensing of asbestos and silica. Science, 320: 674-677. DOI: 10.1126/science. 1156995

Galffy, G., K.A. Mohammed, P.A. Dowling, N. Nasreen and M.J. Ward et al., 1999. Interleukin 8: An autocrine growth factor for malignant mesothelioma. Cancer Res., 59: 367-371.

Garland, L.L., C. Rankin, D.R. Gandara, S.E. Rivkin and K.M. Scott et al., 2007. Phase II study of erlotinib in patients with malignant pleural mesothelioma: A southwest oncology group study. J. Clin. Oncol., 25: 2406-2413. PMID: 17557954 
Govindan, R., R. A. Kratzke, J.E. Herndon II, G.A. Niehans and R. Vollmer et al., 2005. Gefitinib in patients with malignant mesothelioma: A phase II study by the cancer and leukemia group B. Clin. Cancer. Res., 11: 2300-2304. DOI: 10.1158/10780432.CCR-04-1940

Gregorc, V., P. A. Zucali, A. Santoro, G.L. Ceresoli and G. Citterio et al., 2010. Phase II study of asparagine-glycine-arginine-human tumor necrosis factor alpha, a selective vascular targeting agent, in previously treated patients with malignant pleural mesothelioma. J. Clin. Oncol., 28: 2604-2611. PMID: 20406925

Haegens, A., K.J. Butnor, N. Heintz, D. Taatjes and D. Hemenway et al., 2005. Asbestos-induced lung inflammation and epithelial cell proliferation are altered in myeloperoxidase-null mice. Cancer Res., 65: 9670-9677. PMID: 16266986

Hassan, R., S. J. Cohen, M. Phillips, I. Pastan and E. Sharon et al. 2010. Phase I clinical trial of the chimeric anti-mesothelin monoclonal antibody MORAb-009 in patients with mesothelinexpressing cancers. Clin. Cancer Res., 16: 61326138. PMID: 21037025

Hassan, R., W. Ebel, E.L. Routhier, R. Patel and J.B. Kline et al., 2007. Preclinical evaluation of MORAb-009, a chimeric antibody targeting tumorassociated mesothelin. Cancer Immun., 7: 20. PMID: 18088084

Hill, G. D., J.B. Mangum, O.R. Moss and J.I. Everitt, 2003. Soluble ICAM-1, MCP-1 and MIP-2 protein secretion by rat pleural mesothelial cells following exposure to amosite asbestos. Exp. Lung. Res., 29: 277-290. PMID: 12746042

Hillegass, J.M., A. Shukla, S.A. Lathrop, M.B. MacPherson and S.L. Beuschel et al., 2010a. Inflammation precedes the development of human malignant mesotheliomas in a SCID mouse xenograft model. Ann NY Acad Sci., 1203: 7-14. PMID: 20716277

Hillegass, J.M., A. Shukla, M.B. MacPherson, J.P. Bond and C. Steele et al., 2010b. Utilization of gene profiling and proteomics to determine mineral pathogenicity in a human mesothelial cell line (LP9/TERT-1). J. Toxicol. Environ. Health A., 73: 423-436. PMID: 20155583

Jackman, D.M., H.L. Kindler, B.Y. Yeap, P. Fidias and R. Salgia et al., 2008. Erlotinib plus bevacizumab in previously treated patients with malignant pleural mesothelioma. Cancer, 113: 808-814. PMID: 18543326
Janssen-Heininger, Y.M., M.E. Poynter and P.A. Baeuerle, 2000. Recent advances towards understanding redox mechanisms in the activation of nuclear factor kappaB. Free Radic. Biol. Med., 28: 1317-1327. PMID: 10924851

Jube, S. and Z. Rivera, 2012. Cancer cell secretion of the DAMP protein HMGB1 supports progression in malignant mesothelioma. Cancer Res., 72: 32903301. DOI: 10.1158/0008-5472.CAN-11-3481

Kalra, N., J. Zhang, Y. Yu, M. Ho, M. Merino and L. Cao et al., 2012. Efficacy of anti-insulin-like growth factor I receptor monoclonal antibody cixutumumab in mesothelioma is highly correlated with insulin growth factor-I receptor sites/cell. Int. J. Cancer, 131: 2143-2152. DOI: 10.1002/ijc.27471. Epub 2012 Apr 24

Kao, S. C., S. Klebe, D.W. Henderson, G. Reid and M. Chatfield et al. 2011. Low calretinin expression and high neutrophil-to-lymphocyte ratio are poor prognostic factors in patients with malignant mesothelioma undergoing extrapleural pneumonectomy. J. Thorac. Oncol., 6: 1923-1929. PMID: 22011651

Kao, S.C., N. Pavlakis, R. Harvie, J.L. Vardy and M.J. Boyer et al., 2010. High blood neutrophil-tolymphocyte ratio is an indicator of poor prognosis in malignant mesothelioma patients undergoing systemic therapy. Clin. Cancer Res., 16: 58055813. PMID: 20956618

Kelly, R.J., E. Sharon and R. Hassan, 2011. Chemotherapy and targeted therapies for unresectable malignant mesothelioma. Lung. Cancer, 73: 256-263. PMID: 21620512

Klominek, J., B. Baskin, Z. Liu and D. Hauzenberger, et al., 1998. Hepatocyte growth factor/scatter factor stimulates chemotaxis and growth of malignant mesothelioma cells through c-met receptor. Int. J. Cancer, 76: 240-249. PMID: 9537587

Leigh, R.A. and I. Webster 1982. Lymphocytic infiltration of pleural mesothelioma and its significance for survival. S. Afr. Med. J., 61: 10071009. PMID: 7089768

Liu, Z. and J. Klominek, 2004. Chemotaxis and chemokinesis of malignant mesothelioma cells to multiple growth factors. Anticancer, Res., 24: 1625-1630. PMID: 15274332

Lucchi, M., A. Chella, F. Melfi, P. Dini and C. Tibaldi et al., 2007. Four-modality therapy in malignant pleural mesothelioma: A phase II study. J. Thorac. Oncol., 2: 237-242. PMID: 17410047 
Marzo, A.L., D.R. Fitzpatrick, B.W. Robinson B. Scott, 1997. Antisense oligonucleotides specific for transforming growth factor beta2 inhibit the growth of malignant mesothelioma both in vitro and in vivo. Cancer Res., 57: 3200-3207. PMID: 9242450

Mathy, A., P. Baas, O. Dalesio and N.V. Zandwijk 2005. Limited efficacy of imatinib mesylate in malignant mesothelioma: a phase II trial. Lung. Cancer, 50: 83-86. DOI: 10.1016/j.lungcan.2005.04.010

Miserocchi, G. 1997. Physiology and pathophysiology of pleural fluid turnover. Eur. Respir. J., 10: 219225. PMID: 9032518

Moalli, P.A., J.L. MacDonald, L.A. Goodglick and A.B. Kane, 1987. Acute injury and regeneration of the mesothelium in response to asbestos fibers. Am. J. Pathol., 128: 426-445. Mulatero, C.W., R.T. Penson, D. Papamichael, N.H. Gower and M. Evans et al., 2001. A phase II study of combined intravenous and subcutaneous interleukin-2 in malignant pleural mesothelioma. Lung. Cancer, 31: 67-72. PMID: 11162868

Nishimoto, N., 2010. Interleukin-6 as a therapeutic target in candidate inflammatory diseases. Clin. Pharmacol. Ther., 87: 483-487. PMID: 20182422

Ogami, A., Y. Morimoto, H. Yamato, T. Oyabu and T. Kajiwara et al., 2004. Patterns of histopathological change determined by the point counting method and its application for the hazard assessment of respirable dust. Inhal. Toxicol., 16: 793-800.

Oh, S.P., T. Seki, K.A. Goss, T. Imamura and Y. Yi et al., 2000. Activin receptor-like kinase 1 modulates transforminggrowth factor-beta 1 signaling in the regulation of angiogenesis. Proc. Natl. Acad Sci. USA., 97: 2626-2631. PMID: 10716993

Pinato, D.J., F.A. Mauri, R. Ramakrishnan, L. Wahab and T. Lloyd et al., 2012. Inflammation-based prognostic indices in malignant pleural mesothelioma. J. Thorac. Oncol., 7: 587-594. PMID: 22307011

Price, A. 2011. What is the role of radiotherapy in malignant pleural mesothelioma. Oncologist, 16: 359-365. PMID: 21346022

Richards, W.G., L. Zellos, R. Bueno, M.T. Jaklitsch and P.A. Jänne et al., 2006. Phase I to II study of pleurectomy/decortication and intraoperative intracavitary hyperthermic cisplatin lavage for mesothelioma. J. Clin. Oncol., 24: 1561-1567. PMID: 16575008

Rizzardi, C., E. Barresi, A. Brollo, P. Cassetti and M. Schneider et al., 2010. Primary pericardial mesothelioma in an asbestos-exposed patient with previous heart surgery. Anticancer Res., 30: 13231325. PMID: 20530447
Sabo-Attwood, T., M. Ramos-Nino, J. Bond, K.J. Butnor and N. Heintz et al., 2005. Gene expression profiles reveal increased mClca3 (Gob5) expression and mucin production in a murine model of asbestos-induced fibrogenesis. Am. J. Pathol., 167: 1243-1256. PMID: 16251409

Shannahan, J.H., A.J. Ghio, M.C. Schladweiler, J.H. Richards and D. Andrews et al., 2012. Transcriptional activation of inflammasome components by Libby amphibole and the role of iron. Inhal. Toxicol., 24: 60-69. DOI: 10.3109/08958378.2011.633942

Shukla, A., T.F. Barrett, M.B. MacPherson, J.M. Hillegass and N.K. Fukagawa et al., 2011. An extracellular signal-regulated kinase 2 survival pathway mediates resistance of human mesothelioma cells to asbestos-induced injury. Am. J. Respir. Cell Mol. Biol., 45: 906-914. PMID: 21454801

Shukla, A., M. Gulumian, T.K. Hei, D. Kamp and Q. Rahman et al., 2003. Multiple roles of oxidants in the pathogenesis of asbestos-induced diseases. Free Radic. Biol. Med., 34: 1117-1129. PMID: 12706492

Shukla, A., K.M. Lounsbury, T.F. Barrett, J. Gell and M. Rincon et al., 2007. Asbestos-induced peribronchiolar cell proliferation and cytokine production are attenuated in lungs of protein kinase C-delta knockout mice. Am. J. Pathol., 170: 140151. PMID: 17200189

Shukla, A., M. B. MacPherson, J. Hillegass, M.E. Ramos-Nino and V. Alexeeva et al., 2009. Alterations in gene expression in human mesothelial cells correlate with mineral pathogenicity. Am. J. Respir. Cell Mol. Biol., 41: 114-123. PMID: 19097984

Sterman, D. H., A. Recio, R.G. Carroll, C.T. Gillespie and A. Haas et al., 2007. A phase I clinical trial of single-dose intrapleural IFN-beta gene transfer for malignant pleural mesothelioma and metastatic pleural effusions: high rate of antitumor immune responses. Clin. Cancer Res., 13: 4456-4466. PMID: 17671130

Sterman, D.H., A. Recio, A.R. Haas, A. Vachani and S.I. Katz et al., 2010. A phase I trial of repeated intrapleural adenoviral-mediated interferon-beta gene transfer for mesothelioma and metastatic pleural effusions. Mol. Ther., 18: 852-860. PMID: 20068553

Strizzi, L., A. Catalano, G. Vianale, S. Orecchia and A. Casalini et al., 2001. Vascular endothelial growth factor is an autocrine growth factor in human malignant mesothelioma. J. Pathol., 193: 468-475. PMID: 11276005 
Suzuki, E., V. Kapoor, H.K. Cheung, L.E. Ling and P.A. DeLong et al., 2004. Soluble type II transforming growth factor-beta receptor inhibits established murine malignant mesothelioma tumor growth by augmenting host antitumor immunity. Clin. Cancer Res., 10: 5907-5918. PMID: 15355924

Suzuki, E., S. Kim, H.K. Cheung, M.J. Corbley and X. Zhang et al., 2007. A novel small-molecule inhibitor of transforming growth factor beta type I receptor kinase (SM16) inhibits murine mesothelioma tumor growth in vivo and prevents tumor recurrence after surgical resection. Cancer Res., 67: 2351-2359. PMID: 17332368

Suzuki, K., K. Kadota, C.S. Sima, M. Sadelain and V.W. Rusch et al., 2011. Chronic inflammation in tumor stroma is an independent predictor of prolonged survival in epithelioid malignant pleural mesothelioma patients. Cancer Immunol. Immunother., 60: 1721-1728. PMID: 21769693

Takigawa, N., K. Kiura and T. Kishimoto, 2011. Medical treatment of mesothelioma: Anything new? Curr. Oncol. Rep., 13: 265-271. PMID: 21503595

Teta, M.J., E. Lau, B.K. Sceurman and M.E. Wagner, 2007. Therapeutic radiation for lymphoma: Risk of malignant mesothelioma. Cancer, 109: 1432-1438. PMID: 17315168

Travis, L.B., S.D. Fossa, S.J. Schonfeld, M.L. McMaster and C.F. Lynch et al., 2005. Second cancers among 40,576 testicular cancer patients: focus on long-term survivors. J. Natl. Cancer Inst., 97: 1354-1365. PMID: 16174857

Turner, K., S. Varghese and H.R. Alexander, 2012. Current concepts in the evaluation and treatment of patients with diffuse malignant peritoneal mesothelioma. J. Natl. Comprut. Canc. Net., 10: 49-57. PMID: 22223869

Vachani, A., E. Moon and S.M. Albelda, 2011. Gene therapy for mesothelioma. Curr. Treat Options Oncol., 12: 173-180. DOI: 10.1007/s11864-0110153-5
Velcheti, V., Y. Kasai, A.K. Viswanathan, J. Ritter and R. Govindan, 2009. Absence of mutations in the epidermal growth factor receptor (EGFR) kinase domain in patients with mesothelioma. J. Thorac. Oncol., 4: 559. PMID: 19333077

Vogelzang, N.J., J.J. Rusthoven, J. Symanowski, C. Denham and E. Kaukel et al., 2003. Phase III study of pemetrexed in combination with cisplatin versus cisplatin alone in patients with malignant pleural mesothelioma. J. Clin. Oncol., 21: 2636-2644. DOI: $10.1200 /$ JCO.2003.11.136

Yamada, N., S. Oizumi, E. Kikuchi, N. Shinagawa and J.K. Sakakibara et al.. 2010. CD8+ tumorinfiltrating lymphocytes predict favorable prognosis in malignant pleural mesothelioma after resection. Cancer Immunol. Immunother., 59: 1543-1549. PMID: 20567822

Yang, H., M. Bocchetta, B. Kroczynska, A.G. Elmishad and Y. Chen et al., 2006. TNF-alpha inhibits asbestos-induced cytotoxicity via a NF-kappaBdependent pathway, a possible mechanism for asbestos-induced oncogenesis. Proc. Natl. Acad Sci. USA., 103: 10397-10402. DOI: 10.1073/pnas.0604008103

Yang, H., Z. Rivera, S. Jube, M. Nasu and P. Bertino et al., 2010. Programmed necrosis induced by asbestos in human mesothelial cells causes highmobility group box 1 protein release and resultant inflammation. Proc. Natl. Acad Sci. USA., 107: 12611-12616. PMID: 20616036

Zauderer, M.G. and L.M. Krug, 2012. Novel therapies in phase II and III trials for malignant pleural mesothelioma. J. Natl. Comprut. Canc. Net., 10: 42-47. PMID: 22223868

Zha, J. and M.R. Lackner, 2010. Targeting the insulinlike growth factor receptor-1R pathway for cancer therapy. Clin. Cancer Res., 16: 2512-2517. DOI: 10.1158/1078-0432.CCR-09-2232 